Pacific Journal of Mathematics

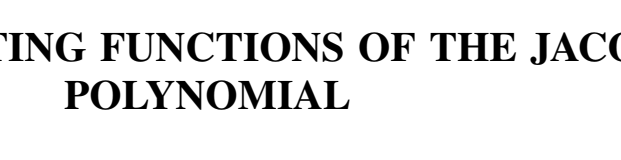




\title{
ON GENERATING FUNCTIONS OF THE JACOBI POLYNOMIALS
}

\author{
Peter Henrici
}

1. Introduction. The series of Jacobi polynomials

$$
\sum_{n=0}^{\infty} a_{n} \rho^{n} P_{n}^{(\nu, \mu)}(\tau)
$$

( $a_{n}$ independent of $\rho$ and $\tau$ ) has in the case $a_{n}=1$ already been evaluated by Jacobi in terms of elementary functions, and there are several other known cases where it can be summed explicity. The sum of (1) is then usually called a generating function of the Jacobi polynomials. On the other hand, according to a particular case of a theorem which we have proved recently, every function of a certain class of regular solutions of the partial differential equation

$$
\frac{\partial^{2} u}{\partial x^{2}}+\frac{\partial^{2} u}{\partial y^{2}}+\begin{gathered}
2 \mu+1 \\
x
\end{gathered} \frac{\partial u}{\partial x}+\begin{gathered}
2 \nu+1 \\
y
\end{gathered} \frac{\partial u}{\partial y}=0
$$

can be represented by a series of type (1), where

$$
\begin{aligned}
& \rho=x^{2}+y^{2}, \\
& \tau=x^{2}-y^{2} \\
& x^{2}+y^{2}
\end{aligned},
$$

and may therefore be considered as a generating function of the Jacobi polynomials in the above sense. This fact is used in the present paper for the construction of an expansion of type (1) which contains several known results of this kind as special cases. As a side result we shall obtain some identities of Cayley-Orr type between the coefficients in the Taylor expansions of certain products of hypergeometric series.

In what follows $x$ and $y$ are considered as independent complex variables. Also the variables

$$
z=x+i y, \quad z^{*}=x-i y
$$

will be used. Our notation of special functions is in accordance with [5].

2. The expansion theorem. The special case $k=0$ of the main theorem of [6] is as follows:

Received June 9, 1954. This paper was prepared under a National Bureau of Standards contract with American University, Washington, D. C. with sponsorship of the Office of Naval Research. 
Theorem. Let

$$
\mu+\nu \neq-2,-3,-4, \cdots
$$

Let

$$
u(x, y)=U\left(z, z^{*}\right)
$$

be a solution of (2) regular in the domain $\mathscr{P}:|z|<r, \quad\left|z^{*}\right|<r \quad(r>0)$ satisfying the conditions

$$
U\left(z, z^{*}\right)=U\left(-z,-z^{*}\right)=U\left(z^{*}, z\right)
$$

and let

$$
U(z, 0)=\sum_{n=0}^{\infty} a_{n} z^{2 n} .
$$

Then $u(x, y)$ has in $\mathscr{P}$ the representation

$$
u(x, y)=\sum_{n=0}^{\infty} \gamma_{n} a_{n} \rho^{n} P_{n}^{(\nu, \mu)}(\tau)
$$

where $\rho$ and $\tau$ are given by (3) and

$$
\gamma_{n}=\frac{2^{2 n} n !}{(\mu+\nu+1+n)_{n}}=\frac{(\mu+\nu+1)_{n} n !}{\left(\begin{array}{c}
\mu+\nu+1 \\
2
\end{array}\right)_{n}\left(\begin{array}{c}
\mu+\nu+2 \\
2
\end{array}\right)_{n}}
$$

3. A special solution of (2). We substitute in (2) bipolar coordinates $(\xi, \eta)$ which we define by

$$
x=\frac{\sinh \xi}{\cosh \xi+\cos \eta}, \quad y=\frac{\sin \eta}{\cosh \xi+\cos \eta} .
$$

They are connected with $\left(z, z^{*}\right)$ and $(\rho, \tau)$ respectively by the relations

$$
\begin{gathered}
\cosh \xi=\underset{\widetilde{\omega}}{1+z z^{*}}=\frac{1+\rho}{\widetilde{\omega}}, \\
\cos \eta=\underset{\widetilde{\omega}}{1-z z^{*}}=\frac{1-\rho}{\widetilde{\omega}},
\end{gathered}
$$

where

$$
\widetilde{\omega}=\sqrt{\left(1-z^{2}\right)\left(1-z^{* 2}\right)}=\sqrt{ } 1-\overline{2 \rho \tau+\rho^{2}} .
$$

(The square roots are positive for $z=z^{*}=0, \rho=0$.) Since (2) may be written in the form

$$
\operatorname{div}\left(x^{2 \mu+1} y^{2 \nu+1} \operatorname{grad} u\right)=0
$$


and since the transformation (10) is isothermal ', we obtain for $\psi(\xi, \gamma)=$ $u(x, y)$ the equation

$$
\frac{\partial}{\partial \xi}\left(x^{2 \mu+1} y^{2 \nu+1} \frac{\partial \psi}{\partial \xi}\right)+{ }_{\partial \eta}^{\partial}\left(x^{2 \mu+1} y^{2 \nu+1} \frac{\partial \psi}{\partial \eta}\right)=0 .
$$

Setting

$$
s=\cosh \xi, \quad t=\cos \eta
$$

and

$$
\psi(\xi, \eta)=(s+t)^{\mu+\nu+1} S(s) T(t)
$$

one finds by the usual separation method that both $S(s)$ and $T(t)$ have to satisfy the differential equation

$$
\left(v^{2}-1\right) \frac{d^{2} V}{d v^{2}}+2(\lambda+1) v^{d V} d v+[\lambda(\lambda+1)-\kappa(\kappa+1)] V=0,
$$

where $v=s, \lambda=\mu$, if $V=S$, and $v=t, \lambda=\nu$, if $V=T, k$ being a separation parameter. A solution of (14) regular near $v=1$ is the function

$$
\begin{aligned}
V_{\kappa}^{\lambda}(v) & ={ }_{2} F_{1}\left[\begin{array}{c}
\lambda-\kappa, \lambda+\kappa+1 ; \\
\lambda+1
\end{array}\right] \\
& =I(\lambda+1)\left(v^{2}-1\right)^{-\lambda / 2} P_{\kappa}^{-\lambda}(v) .
\end{aligned}
$$

Here $P$ denotes the Legendre function of the first kind. ${ }^{2}$ Tracing back our substitutions and assuming that none of the numbers $\mu$ and $\nu$ is a negative integer, we may thus define a solution of (2) by

$$
\Phi_{\kappa}^{(\mu, \nu)}(\rho, \tau)=\widetilde{\omega}^{-\mu-\nu-1} V_{\kappa}^{\mu}\left(\frac{1+\rho}{\widetilde{\omega}}\right) V_{\kappa}^{\nu}\left(\frac{1-\rho}{\widetilde{\omega}}\right) .
$$

Evidently this function satisfies the functional relations

$$
\begin{aligned}
& \Phi_{\kappa}^{(\mu, \nu)}(\rho, \tau)=\Phi_{-\kappa-1}^{(\mu, \nu)}(\rho, \tau) \\
& \Phi_{\kappa}^{(\mu, \nu)}(\rho, \tau)=\Phi_{\kappa}^{(\nu, \mu)}(-\rho,-\tau) .
\end{aligned}
$$

Among the many possible representations of $\Phi_{\kappa}^{(\mu, \nu)}$ in terms of hypergeometric functions we list the following, which is obtained by substituting equation 3.2 (24) of [5] for the Legendre functions involved in (15):

${ }^{2}$ The functions $V_{\kappa} \lambda(v)$ could also be expressed in terms of Gegenbauer functions. 


$$
\begin{aligned}
& \Phi_{\kappa}^{(\mu, \nu)}(\rho, \tau)=\varphi_{2} F_{1}\left[\begin{array}{cl}
\mu+\kappa+1 & \frac{\mu+\kappa+2}{2} \\
2 & \mu+1
\end{array}\right] \\
& \times{ }_{2} F_{1}\left[\frac{\nu-\kappa}{2}, \frac{\nu-\kappa+1}{2} ; Y\right] \text {. }
\end{aligned}
$$

Here we have put

$$
\varphi=(1+\rho)^{-\mu-\kappa-1}(1-\rho)^{\kappa-\nu}=\left(1+z z^{*}\right)^{-\mu-\kappa-1}\left(1-z z^{*}\right)^{\kappa-\nu}
$$

and

$$
\begin{aligned}
& X=\frac{2 \rho(\tau+1)}{(1+\rho)^{2}}=\left(\begin{array}{c}
z+z^{*} \\
1+z z^{*}
\end{array}\right)^{2}, \\
& Y=\frac{2 \rho(\tau-1)}{(1-\rho)^{2}}=\left(\frac{z-z^{*}}{1-z z^{*}}\right)^{2} .
\end{aligned}
$$

It is easy to see from this representation that the function

$$
U\left(z, z^{*}\right)=\Phi_{\kappa}^{(\mu, \nu)}(\rho, \tau)
$$

is regular in $|z|<1,\left|z^{*}\right|<1$ and that it satisfies the symmetry relations (6). Save for the mentioned exceptional values of the parameters, (15) defines therefore a solution of (2) for which the assumptions of the expansion principle of $\S 2$ are satisfied.

4. The Jacobi expansion of $\Phi_{\kappa}^{(\mu, \nu)}$. From (18) we have immediately

$$
U(z, 0)={ }_{2} F_{1}\left[\frac{\mu+\kappa+1}{2}, \frac{\mu+\kappa+2}{2} ; z^{2}\right]_{2} F_{1}\left[\frac{\nu-\kappa}{2}, \frac{\nu-\kappa+1}{2} ; z^{2}\right] .
$$

If we denote by $a_{n}$ the coefficient of $z^{2 n}$ in the Taylor expansion of the right hand side of (19), we obtain by the expansion principle the series

$$
\widetilde{\omega}^{-\mu-\nu-1} V_{\kappa}^{\mu}\left(\frac{1+\rho}{\widetilde{\omega}}\right) V_{\kappa}^{\nu}\left(\frac{1-\rho}{\widetilde{\omega}}\right)=\sum_{n=0}^{\infty} \gamma_{n} a_{n} \rho^{n} P_{n}^{(\nu, \mu)}(\tau),
$$

which converges if

$$
|z|<1, \quad\left|z^{*}\right|<1,
$$

or, what amounts to the same,

$$
\left|\rho\left(\tau \pm \sqrt{\tau^{2}-1}\right)\right|<1 .
$$

We note the following representations of $a_{n}$ in terms of terminating hypergeometric series: 
(21) $\quad a_{n}=\frac{\left(\frac{\nu-\kappa}{2}\right)_{n}\left(\frac{\nu-\kappa+1}{2}\right)_{n}}{(\nu+1)_{n} n !}{ }_{4} F_{3}$

$$
\left[\begin{array}{ccc}
\mu+\kappa+1 & \mu+\kappa+2 \\
2 & , & -\nu-n,-n ; \\
\mu+1, & \frac{2-\nu+\kappa}{2}-n, & \frac{1-\nu+\kappa}{2}-n
\end{array}\right]
$$

$$
\begin{aligned}
& a_{n}=\gamma_{n}^{-1} \frac{(\nu-\kappa)_{n}{ }_{3} F_{2}}{(\nu+1)_{n}}\left[\begin{array}{l}
\mu+\kappa+1, \kappa+1,-n ; \\
\mu+1,1+\kappa-\nu-n
\end{array}\right], \\
& a_{n}=\gamma_{n}^{-1} \begin{array}{cc}
(\mu+\nu+1)_{n}{ }_{3} F_{2} \\
(\nu+1)_{n}
\end{array}\left[\begin{array}{ll}
\mu+\kappa+1, & \mu-\kappa,-n ; \\
\mu+1, & \mu+\nu+1
\end{array}\right] .
\end{aligned}
$$

Of these, (21) is obtained by straightforward Cauchy multiplication of the two power series on the right of (19). In order to prove (22), we consider (20) for the special value $\tau=1$ (that is, $z=z^{*}$ ). This gives on the left, using (17),

$$
U(z, z)=\left(1+z^{2}\right)^{-\mu-\kappa-1}\left(1-z^{2}\right)^{\kappa-\nu}{ }_{2} F_{1}\left[\begin{array}{ccc}
\mu+\kappa+1 & \mu+\kappa+2 & 4 z^{2} \\
2 & 2 & \left(1+z^{2}\right)^{2}
\end{array}\right] .
$$

By a quadratic transformation [5, eq. 2.11 (34)] this ${ }_{2} F_{1}$ can be expressed by one with argument $z^{2}$, and in view of

$$
P_{n}^{(\nu, \mu)}(1)=\frac{(\nu+1)_{n}}{n !}
$$

(20) thus becomes

$$
\left(1-z^{2}\right)^{\kappa-\nu}{ }_{2} F_{1}\left[\begin{array}{c}
\mu+\kappa+1, \kappa+1 ; z^{2} \\
\mu+1
\end{array}\right]=\sum_{n=0}^{\infty} \gamma_{n} a_{n} \frac{(\nu+1)_{n}}{n !} z^{2 n} .
$$

From this (22) follows again by Cauchy multiplication of the series on the left. Putting $\tau=-1$ (or $z=-z^{*}$ ) in (20) leads in a similar way to

$$
\left(1--z^{2}\right)^{\kappa-\mu}{ }_{2} F_{1}\left[\begin{array}{c}
\nu+\kappa+1, \kappa+1 ; z^{2} \\
\nu+1
\end{array}\right]=\sum_{n=0}^{\infty} \gamma_{n} a_{n} \begin{gathered}
(\mu+1)_{n} \\
n !
\end{gathered} z^{2 n}
$$

The representation (23) of $a_{n}$ is remarkable for the fact that only one parameter in the ${ }_{3} F_{2}$ depends on $n$. It is obtained by expressing the hypergeometric function on the left of (25) by one with argument $z^{2} /\left(z^{2}-1\right)$, expanding in terms of this argument, expanding the powers of $z^{2} /\left(z^{2}-1\right)$ in terms of powers of $z^{2}$ and rearranging.

From (19) it is easily seen by applying Euler's linear transformation to the two hypergeometric series simultaneously that $U(z, 0)$ and hence $a_{n}$ is a symmetric function of $\mu$ and $\nu$. Therefore in (21), (22) and (23) the variables $\mu$ and $\nu$ may be interchanged. Furthermore, in view of (17) $\kappa$ may be replaced everywhere by $-\kappa-1$. Many other 
representations for the coefficients $a_{n}$ could be derived from the ones given above by the application of transformations of generalized hypergeometric series of unit argument. One example for this technique will be given at the end of $\S 6$.

5. Special cases. (i) If $\kappa=0$, (23) yields by Vandermonde's theorem

$$
a_{n}=\gamma_{n}^{-1}(\mu+\nu+1)_{n} F_{1}\left[\begin{array}{c}
\mu,-n ; \\
\mu+\nu+1
\end{array}\right]=\gamma_{n}^{-1}
$$

and from (15) we have

$$
V_{0}^{\lambda}(v)=\left(\begin{array}{c}
1+v \\
2
\end{array}\right)^{-\lambda}
$$

Thus (20) reduces to

$$
2^{\mu+\nu} \widetilde{\omega}^{-1}(1+\rho+\widetilde{\omega})^{-\mu}(1-\rho+\widetilde{\omega})^{-\nu}=\sum_{n=0}^{\infty} \rho^{n} P_{n}^{(\nu, \mu)}(\tau) .
$$

This is the classical generating series of $\mathrm{Jacobi}^{3}$.

(ii) Since

$$
V_{\lambda}^{\lambda}(v)=1,
$$

other noteworthy special cases of (20) are to be expected for $\kappa=\mu$ or $\kappa=\nu$. In the first case we have from (23) (using the symmetry with respect to $\mu$ and $\nu$ )

$$
a_{n}=\gamma_{n}^{-1}-(\mu+\nu+1)_{n} .
$$

Thus (20) yields, if (18) is used on the left,

$$
\begin{aligned}
& (1+\rho)^{-\mu-\nu-1}{ }_{2} F_{1}\left[\frac{\mu+\nu+1}{2}, \frac{\mu+\nu+2}{2} \underset{\mu+1}{2} ; \frac{2 \rho(\tau+1)}{(1+\rho)^{2}}\right] \\
& =\sum_{n=0}^{\infty} \frac{(\mu+\nu+1)_{n}}{(\mu+1)_{n}} \rho^{n} P_{n}^{(\nu, \mu)}(\tau) \text {. }
\end{aligned}
$$

An equivalent formula is easily derived from a bilinear generating function due to Watson [10] and has been stated explicitly (but with a slight algebraic error) by Bailey [1, p. 102]. The result is given correctly by Buchholz [3, p. 143. eq. (20)].

The case $k=\nu$ does in view of (17) not lead to something new. A 
similar, but not equivalent formula can be deduced from (20) by putting $\kappa=\mu+1$ or $\kappa=\nu+1$.

If $\kappa=\mu=\nu$, we obtain from (20) and (29) in virtue of (28) and

$$
P_{n}^{(\mu, \mu)}(\tau)=\frac{(\mu+1)_{n}}{(2 \mu+1)_{n}} C_{n}^{\mu+1 / 2}(\tau),
$$

the classical generating series of the Gegenbauer polynomials

$$
\widetilde{\omega}^{-2 \mu-1}=\sum_{n=0}^{\infty} \rho^{n} C_{n}^{\mu+1 / 2}(\tau) .
$$

(iii) Also in the cases $\mu= \pm \frac{1}{2}$ (or $\nu= \pm \frac{1}{2}$ ) the Jacobi polynomials reduce to Gegenbauer polynomials. Since $\Phi_{\kappa}^{(\mu, \nu)}$ likewise may be expressed in terms of Gegenbauer functions, (20) takes then the form of an addition theorem for these functions. This result has been given by us already elsewhere [7].

(iv) Putting $\rho=r / \kappa^{2}$ and letting $k \rightarrow \infty$, we obtain from (20) and (23), since

$$
\lim _{\kappa \rightarrow \infty} V_{\kappa}^{\lambda}\left(1-2 w / \kappa^{2}\right)={ }_{0} F_{1}[\lambda+1 ; w],
$$

the well-known formula (see the references to equation (42) of [6])

$$
{ }_{0} F_{1}\left[\mu+1 ; r \frac{\tau+1}{2}\right]{ }_{0} F_{1}\left[\nu+1 ; r \frac{\tau-1}{2}\right]=\sum_{n=0}^{\infty}(\mu+1)_{n}(\nu+1)_{n} P_{n}^{(\nu, \mu)}(\tau) .
$$

With the exception of a result of Brafman [2], the special cases of (20) mentioned above cover to our knowledge all simple (that is, not bilinear) known generating functions of the Jacobi polynomials which are valid for general values of $\mu$ and $\nu .{ }^{4}$

6. Identities of Cayley-Orr type. The formulae (19), (25) and (26) suggest identities between the coefficients of the expansions of certain hypergeometric products which in a symmetric way may be stated as follows :

Each of the three identities

$$
\begin{aligned}
& (1-\zeta)^{\kappa-\nu}{ }_{2} F_{1}\left[\begin{array}{c}
\mu+\kappa+1, \kappa+1 ; \zeta \\
\mu+1
\end{array}\right]=\sum_{n=0}^{\infty}(\nu+1)_{n} A_{n} \zeta^{n}, \\
& (1-\zeta)^{\kappa-\mu}{ }_{2} F_{1}\left[\begin{array}{c}
\nu+\kappa+1, \kappa+1 ; \zeta \\
\nu+1
\end{array}\right]=\sum_{n=0}^{\infty}(\mu+1)_{n} A_{n} \zeta^{n},
\end{aligned}
$$

4 Brafman's result, which was originally established as a corollary to Bailey's decomposition formula for Appell's function $F_{4}$, has been proved by our method without the use of Bailey's formula in [6]. 


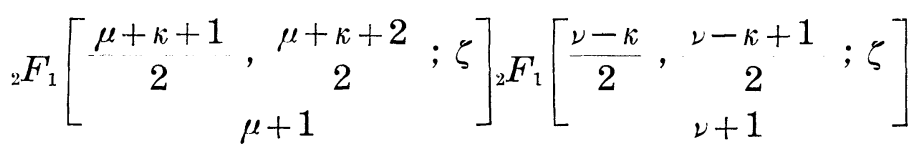

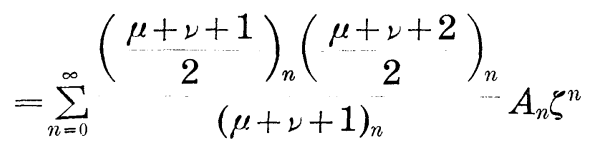

implies the other two.

This result is of a type considered first by Cayley and Orr [8]. While (a) $\sim(b)$ is a special case of a result by Burchnall and Chaundy (see [4, eq. (13)]), the two equivalencies (a) (c) and (b) $(\mathrm{c})$ as well as the method of their derivation seem to be new. Identities of this type have been investigated either by a discussion of the ordinary differential equations satisfied by the products of hypergeometric functions (for recent results obtained by this method, see [4]) or by transformations of the generalized hypergeometric series arising in the Cauchy multiplication of the power series under consideration. An account of Bailey's and Whipple's work in this direction can be found in [1]. In order to render our above result independent of the consideration of a special partial differential equation, we sketch a short proof of it by Whipple's method. By reasons of symmetry it suffices to prove (a) (c). This amounts to a direct proof of the equality of (21) and (22). We first transform the ${ }_{3} F_{2}$ in (22) into a saalschützian ${ }_{4} F_{3}$ by equation 4.5 (1) of [1]. This gives

$$
\begin{aligned}
& \gamma_{n} a_{n}=\frac{(\nu-\kappa)_{n}}{(\nu+1)_{n}} F_{2}\left[\begin{array}{l}
\mu+\kappa+1, \kappa+1,-n ; \\
\mu+1,1+\kappa-\nu-n
\end{array}\right] \\
& =\underset{(\nu+1)_{n}}{(\mu+\nu+1)_{n-1} F_{3}}\left[\begin{array}{ccc}
\mu+\kappa+1 & , \frac{\mu-\kappa}{2}, \mu+\nu+1+n,-n ; \\
2 & 2 & \mu \\
\mu+1, & \mu+\nu+1 & \mu+\nu+2 \\
2 & 2
\end{array}\right] \text {. }
\end{aligned}
$$

The desired result is now established by transforming the ${ }_{4} F_{3}$ according to equation 7.2 (1) of [1]. We emphasize that it is also possible to prove (a) (c) by the differential equation method of Burchnall and Chaundy.

\section{REFERENCES}

1. W. N. Bailey, Generalized hypergeometric series, Cambridge 1935.

2. F. Brafman, Generating functions of Jacobi and related polynomials, Proc. Amer. Math. Soc., 2 (1951), 942-949.

3. H. Buchholz, Die konfluente hypergeometrische Funltion, Berlin, Göttingen, Heidelberg 1953. 
4. J. W. Burchnall and T. W. Chaundy, The hypergeometric identies of Cayley, Or'r, and Bailey, Proc. Lond. Math. Soc., (2) 50 (1948-49), 56-74.

5. A. Erdélyi, W. Magnus, F. Oberhettinger and F. Tricomi, Higher transcendental functions, vols. I \& II, New York 1953.

6. P. Henrici, On certain series expansions involving Whittaker functions and Jacobi polynomials, Pacific J. of Math., 5 (1955), 725-744.

7. _._..., Ueber die Funktionen von Gegenbauer, Archiv der Mathematik, 5 (1954), 92-98.

8. W. McF. Orr, Theorems relating to the product of tuo hypergeometric serics, Trans. Camb. Phil. Soc., 17 (1899), 1-15.

9. G. Szegö, Orthogonal polynomials, New York 1939.

10. G. N. Watson, Notes on generating functions of polynomials: (4) Jacobi polynomials, J. Lond. Math. Soc., 9 (1934), 22-28.

11. F. J. W. Whipple, On a formula implied in Orr's theorem concerning the products of hypergeometric series, J. Lond. Math. Soc., 4 (1929), 48-50.

National Bureau of Standards

Added in proof: Without giving details we mention an interesting "confluent" case of the generating function (20). This is obtained by setting

$$
\tau=\frac{2 x}{\nu}-1, \quad k^{2}=-v y
$$

$(x, y$ fixed) and letting $\nu \rightarrow \infty$. The result is the well-known series (see [9], p. 98)

$$
\sum_{n=0}^{\infty} \frac{(-)^{n} n !}{(\mu+1)_{n}} L_{n}{ }^{(\mu)}(x) L_{n}{ }^{(\mu)}(y) \rho^{n}=(1+\rho)^{-\mu-1} \exp \frac{\rho(x+y)}{1+\rho}{ }_{0} F_{1}\left[\mu+1 ;-\frac{x y \rho}{(1+\rho)^{2}}\right],
$$

where $L_{n}^{(\mu)}$ denotes the Laguerre polynomial. 

.././. ./FrontMatter/paper .pdf 


\section{Pacific Journal of Mathematics}

Nesmith Cornett Ankeny and Theodore Joseph Rivlin, On a theorem of S.

Bernstei........................................ 849

Louis Auslander, The use of forms in variational calculation .......... 853

Paul Civin, Abstract Riemann sum . .......................... 861

Paul Civin, Some ergodic theorems involving two operator ............ 869

Eckford Cohen, The number of solutions of certain cubic congruence .... . 877

Richard M. Cohn, Specializations over difference field .............. 887

Jean Dieudonné, Pseudo-discriminant and Dickson invarian . . ......... 907

Ky Fan, A comparison theorem for eigenvalues of normal matrice ........ 911

Richard P. Gosselin, On the convergence behaviour of trigonometric interpolating polynomial ........................... 915

Peter K. Henrici, On generating functions of the Jacobi polynomial . . . . . . . 923

Meyer Jerison, An algebra associated with a compact grou ............ 933

Wilhelm Magnus, Infinite determinants associated with Hill's equatio . . . . . 941

G. Power and D. L. Scott-Hutton, The slow steady motion of liquid past a semi-elliptical bos................................. 953

Lyle E. Pursell, An algebraic characterization of fixed ideals in certain function ring .................................... 963

C. T. Rajagopal, Additional note on some Tauberian theorems of O. Szás . . 971 Louis Baker Rall, Error bounds for iterative solutions of Fredholm integral

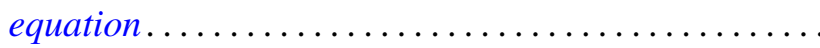

Shigeo Sasaki and Kentaro Yano, Pseudo-analytic vectors on

pseudo-Kählerian manifold ......................

Eugene Schenkman, On the tower theorem for finite group

P. Stein and John E. L. Peck, On the numerical solution of Poisson's equation over a rectangl ........................

Morgan Ward, The mappings of the positive integers into themselves which preserve divisio .

Seth Warner, Weak locally multiplicatively-convex algebra 1025

Louis Weisner, Group-theoretic origin of certain generating function .... 